\title{
Light-sheet microscopy using MEMS and active optics for 3D image acquisition control
}

\author{
Spyridon Bakas*, Deepak Uttamchandani, Ralf Bauer \\ Centre of Microsystems \& Photonics, Department of Electronic \& Electrical Engineering, \\ University of Strathclyde, Glasgow G1 1XW, United Kingdom
}

\begin{abstract}
A miniaturized version of a light-sheet microscopy (LSM) system, with 3D imaging enabled through active optical control, is presented. Even though the field of LSM technology has advanced significantly in recent years, it is still not considered an easily available technique. This is mainly due to its cost compared to epifluorescence setups and the requirement for specific sample mounting techniques in most cases, as well as stringent optical alignment and difficulty to reduce motion artifacts when the sample is moved through the light path to create the imaging slices. In our research, we demonstrate a miniaturized version of an LSM that can reduce size and cost, and is able to achieve 3D imaging through control of multiple active optical elements and MEMS micromirrors used in both the illumination and imaging path instead of moving the sample. The laser excitation is controlled and shaped via multiple MEMS elements for 3D beam position control and multilens beam shaping to generate a $2.85 \mu \mathrm{m}$ wide light-sheet with controllable height of up to $550 \mu \mathrm{m}$, and orthogonal positioning over a $200 \mu \mathrm{m}$ range. Additionally, the focal point of the excitation can be shifted along the laser propagation direction by $200 \mu \mathrm{m}$. The orthogonally positioned imaging path incorporates a x20, NA $=0.4$ objective and a tunable lens for imaging selected focal planes synchronized with the excitation positioning. The imaging results show sub-micron resolution with a field-of-view of $400 \mu \mathrm{m}$ x $300 \mu \mathrm{m}$. The synchronization of the two active elements allows for fast imaging of different slices of a sample and promises convenient 3D reconstruction and representation of cell tissue.
\end{abstract}

Keywords: MEMS micromirror, light-sheet microscopy, miniaturized imaging, fluorescence imaging

\section{INTRODUCTION}

A crucial factor of advancing biomedical science is the ability to utilize imaging tools that will allow detailed and highspeed imaging of biological processes without adversely affecting the samples. Epifluorescence microscopy is the leading technique in the field due to high quality contrast imaging, easy imaging setups and wide availability of widefield microscopes which can be directly fitted for Epifluorescence imaging, but suffer from severe limitations as the sample can lose its ability to fluoresce over short time periods (photobleaching) or cells can be permanently damaged due to laser light exposure (phototoxicity) ${ }^{1}$. An alternative to classic fluorescence microscopy methods (e.g. epifluorescence microscopy, confocal microscopy) for fast three-dimensional fluorescence imaging is Light-sheet Microscopy (LSM) ${ }^{2,3,4}$. In LSM only a thin sheet of light illuminates the sample and the illuminated slice is being imaged orthogonally, reducing unintended fluorescence excitation of the rest of the sample (compared to epifluorescence microscopy) and enabling higher speed of imaging (compared to confocal microscopy). By either axially moving or rotating the sample, these slices can be combined to represent a 3D image ${ }^{5,6}$. The field of LSM microscopy research evolves in recent times towards new methods ${ }^{7}$ that attempt to explore super resolution microscopy ${ }^{8}$ with added features targeting light-sheet uniformity ${ }^{9,10}$ and the use of specialized beams ${ }^{11,12}$. As a result systems tend to be more expensive and not easily accessible to research labs worldwide. Recent studies have provided interesting results on making LSM systems more accessible through size improvements ${ }^{13}$ or open access protocols ${ }^{14}$. In our research we are introducing a miniaturized version of an LSM designed with of-the-shelf optics and custom 3D printed features reducing total size and cost of manufacture. Additionally, we enable further control of the illumination and imaging path through active optical elements included in both arms of the microscope. Control of the illumination path in both the direction of beam and image propagation is achieved with an electrothemal varifocal Microelectromechancial Systems micromirror (VFM MEMS) and a piezoelectric scanning MEMS micromirror (PZT MEMS) respectively. Furthermore, an electrical tunable lens is used to address focal plane changes in the imaging path during imaging of samples fixed at microscope slides. 


\section{SYSTEM CHARACTERISTICS AND DESIGN}

\subsection{LSM system design}

The top view schematic for the miniaturized LSM is shown in Figure 1. A solid state laser with $\lambda=473 \mathrm{~nm}$ is fibre coupled and transmitted towards the 45 degree placed VFM mirror. The collimated beam is guided to the PZT MEMS mirror and redirected to enter a $4 \mathrm{f}$ lens system to increase the beam diameter at the back-aperture of a focusing lens placed in a telecentric setup. The system consisted of 3 achromatic lens doublets with $\mathrm{f}=7.5 \mathrm{~mm}, \mathrm{f}=30 \mathrm{~mm}$ and $\mathrm{f}=7.5 \mathrm{~mm}$. The imaging path is built along the $\mathrm{x}$-axis with a 20x standard microscope objective (Newport MVC-20X, NA =0.4) that will collect the projected section as an image and transmit it towards the sCMOS camera (Quantalux CS2100M-USB sCMOS camera). Additionally, a tunable lens (Optotune EL-3-10) is used to allow synchronisation of focal planes of the sample during imaging. All optical elements used in the system are housed in custom-made 3D printed holders ensuring stability during alignment as well as making use of the limited space resulting from miniaturization.

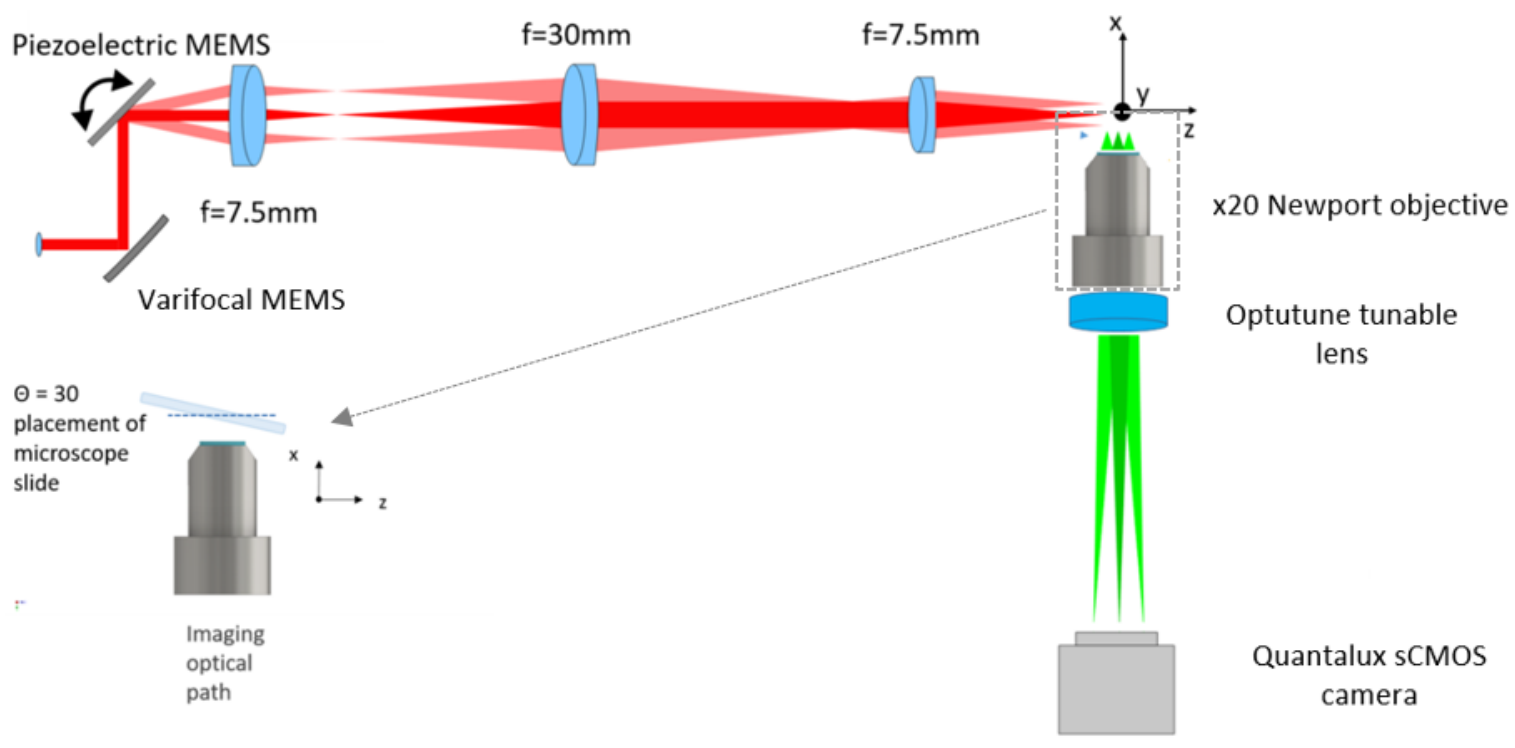

Figure 1. Miniaturized LSM schematic showing the optical beam paths of both excitation and imaging as well as all included optical elements.

\subsection{Varifocal MEMS mirror}

The VFM MEMS mirror in the illumination path is used to enable a focal position change of the light-sheet beam waist along the laser propagation direction. The $1.8 \mathrm{~mm}$ mirror diameter MEMS, shown schematically in Figure 2(c), is fabricated using the SOIMUMPs multi-user fabrication process offered by MEMSCAP Inc. A $10 \mu \mathrm{m}$ silicon-on-insulator layer is used as structural device layer, on which a $500 \mathrm{~nm}$ gold layer is deposited to create the mirror surface. The device is actuated through an electrical current through the mirror surface, which leads to Joule heating and a change of curvature due to the different thermal expansion coefficients of the mirror material layers. The mirror surface is suspended from the substrate using eight serpentine springs on the mirror perimeter. The heat induced change of the curvature corresponds to a respective focal plane change in the reflected optical path.

The curvature change during actuation with an applied voltage of up to $15 \mathrm{~V}$, corresponding to an electrical power up to $130 \mathrm{~mW}$, was measured with a Veeco NT1100 surface profiler and the results are presented in Figure 2(a) and Figure 2(b). The static response of the mirror radius of curvature was found to increase from $35 \mathrm{~mm}$ to $65 \mathrm{~mm}$ during thermal damage limited actuation. Dynamic mirror curvature changes can be achieved when actuating the mirror at its mechanical resonance Eigenfrequency, with two useful mirror modes located at $6 \mathrm{kHz}$ and $38 \mathrm{kHz}$ based on structural simulations done in Comsol Multiphysics. 

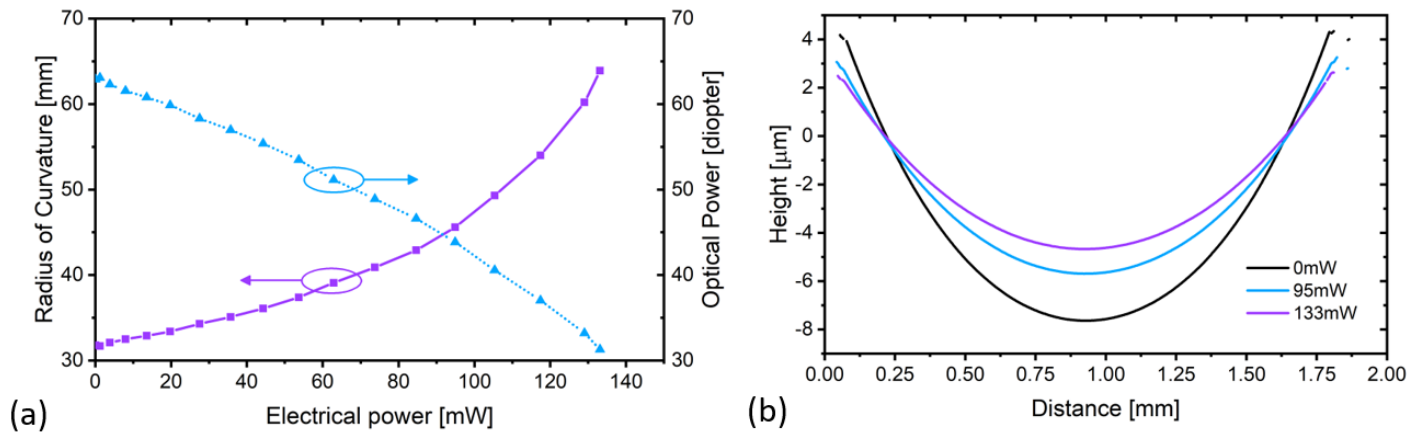

(c)

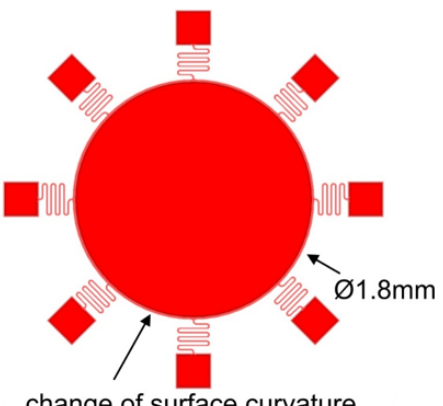

change of surface curvature

Figure 2. (a) VFM MEMS characterization of Radius of curvature (ROC) versus the applied electrical power (b) Cross-section of VFM mirror surface for 3 actuation cases (c) Top-view schematic of VFM MEMS mirror

\subsection{Scanning MEMS mirror}

Formation and orthogonal repositioning of the light-sheet is achieved with the use of a 2D scanning MEMS mirror, shown schematically in Figure 3(a). The mirror has two distinct movement axes, of which one can be resonantly actuated due to a PZT layer on the inner ring frame, and the other can create static angular displacement. The static angle change is also based on piezoelectric actuation, which will take place in the serpentine actuators connecting the outside of the frame to the substrate and leading to a static movement of the mirror on the vertical axis. The mirror diameter is $1.1 \mathrm{~mm}$, whereas the device is composed of a $100 \mu \mathrm{m}$ thick silicon substrate coated with a $2 \mu \mathrm{m}$ layer of PZT on the actuators surface ${ }^{15}$.
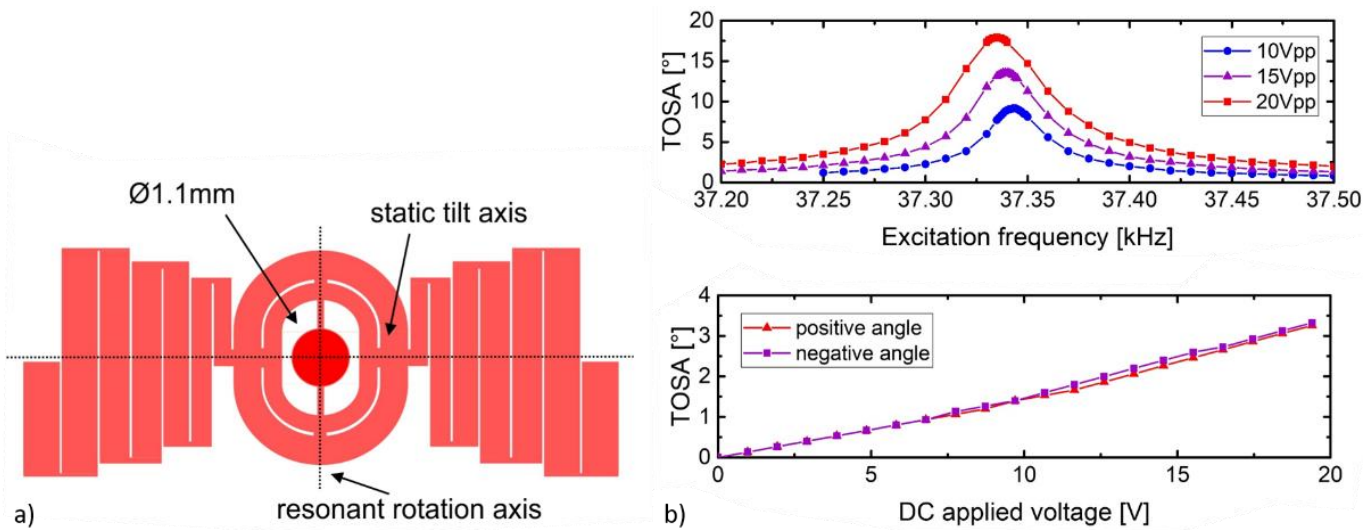

Figure 3. (a) PZT MEMS schematic (b) Total Optical Scan angle (TOSA) characteristic during resonance (c) Static TOSA versus applied voltage.

Three different amplitudes of a unipolar square-wave input voltage signal were used to test the resonance response of the inner ring actuator namely, $10 \mathrm{~V}, 15 \mathrm{~V}$ and $20 \mathrm{~V}$, with optical angles reaching at the maximum resonance point $9.2^{\circ}, 13.5^{\circ}$ and $18^{\circ}$ respectively. The static angle response was recorded for DC input voltages up to $20 \mathrm{~V}$, with a responding angle of 
$3.3^{\circ}$ in both mirror movement directions. The corresponding characterization plots are shown in Figure 3(b) and Figure 3(c).

\subsection{System characterization}

Characterization of the focused beam to generate the LSM excitation was accomplished with an orthogonally positioned test imaging setup, including a x10 standard objective and imaging camera, which images the beam propagation through a rectangular cuvette filled with fluorescein solution. The excitation beam around the focal plane can be seen in Figure 4 for three different static actuations of the piezoelectric mirror, corresponding to three different beam positions on the $\mathrm{x}$ axis. The three positions represent both the maximum static angles of the MEMS and the position of the MEMS when it is unactuated (Figure 4(b), (c) and (a), respectively). The focal point position along the propagation axis was shifted by less than $3 \mu \mathrm{m}$ for actuation between 0 and the maximum angles. The beam diameter in the focal point is measured at $2.84 \mu \mathrm{m}$, whereas the beam diameter remains below $26 \mu \mathrm{m}$ in a range of $-200 \mu \mathrm{m}<\mathrm{z}<200 \mu \mathrm{m}$. The Gaussian beam characteristics can also be seen in Figure 4 for three different selected profile plots; $120 \mu \mathrm{m}$ from the focus (d) $(\mathrm{FWHM}=$ $11.05 \mu \mathrm{m})$, at the focus $(\mathrm{e})(\mathrm{FWHM}=2.84 \mu \mathrm{m})$ and $40 \mu \mathrm{m}$ from the focus $(\mathrm{f})(\mathrm{FWHM}=4.89 \mu \mathrm{m})$. The beam width represents the thickness of the light-sheet on the same axis when operating in the scanned light-sheet mode.
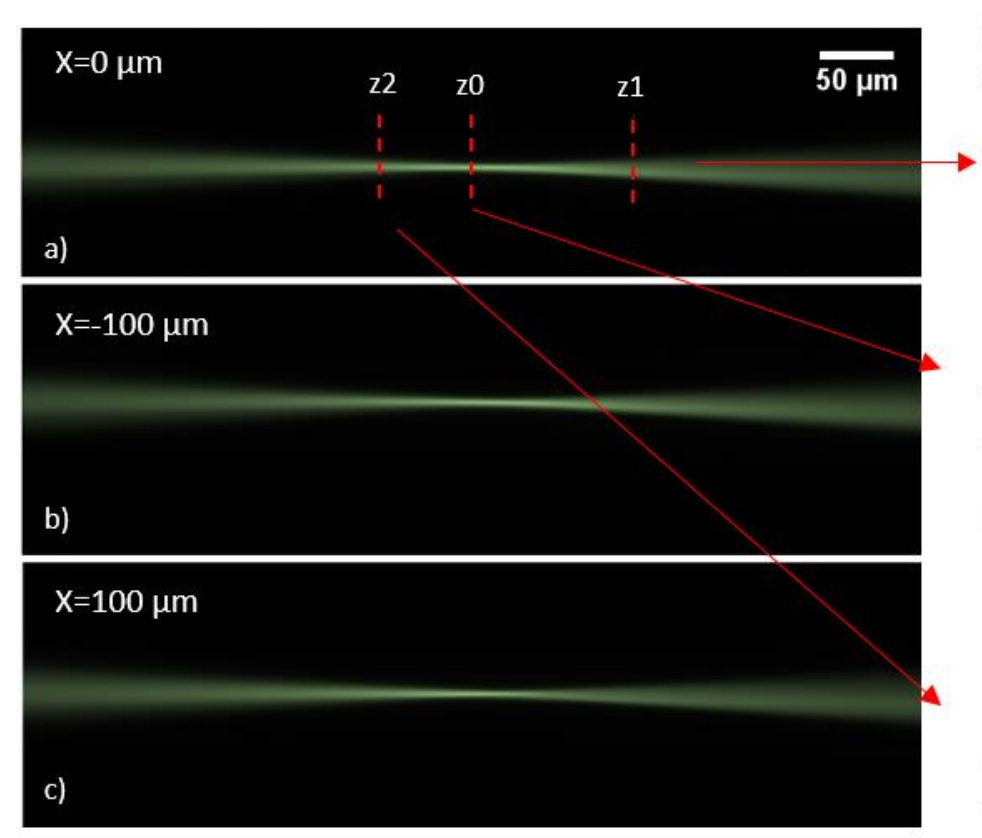
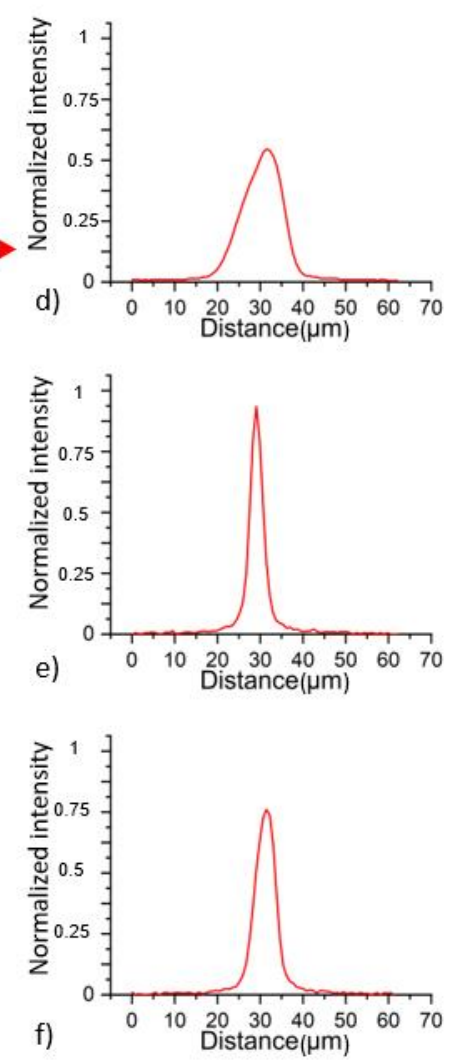

Figure 1. The propagation characteristics for the focused beam are shown in three different positions corresponding to (a) the PZT mirror being unactuated and (b) actuated to the minimum and (c) maximum static angles. Additionally, sections of the beam are plotted for three different positions along the propagation direction namely, (d) $\mathrm{z}_{1}=120 \mu \mathrm{m}$, (e) $\mathrm{z}_{0}=0 \mu \mathrm{m},(\mathrm{f}) \mathrm{z}_{2}=40 \mu \mathrm{m}$.

An important aspect of this LSM setup is the control of the focal plane for the laser beam with the use of the VFM mirror. In this way the focusing point will be actively translated along the propagation direction allowing for thinnest sectioning during imaging. Using a Gaussian beam for excitation, this ability to create the thinnest possible excitation can result in higher contrast in fluorescence images due to lower background excitation. The curvature change of the mirror will result in the beam (or light-sheet) to focus further away from the excitation lens compared to its initial position when the mirror was unactuated. Figure 5(a) shows the focused beam waist with $\mathrm{V}_{\text {varifocal }}=0 \mathrm{~V}$ where the beam is being focused at $5.2 \mathrm{~mm}$ away from the final lens. Actuation of the mirror with $\mathrm{V}_{\text {varifocal }}=15 \mathrm{~V}$ shows that the focus of the beam has been moved 
$200 \mu \mathrm{m}$ away from its original position (Figure 5(b)). In future implementations this change will allow an active synchronization with the rolling shutter of the imaging arm of the microscope in order to always section the sample with the thinnest light-sheet possible over a larger field of view.
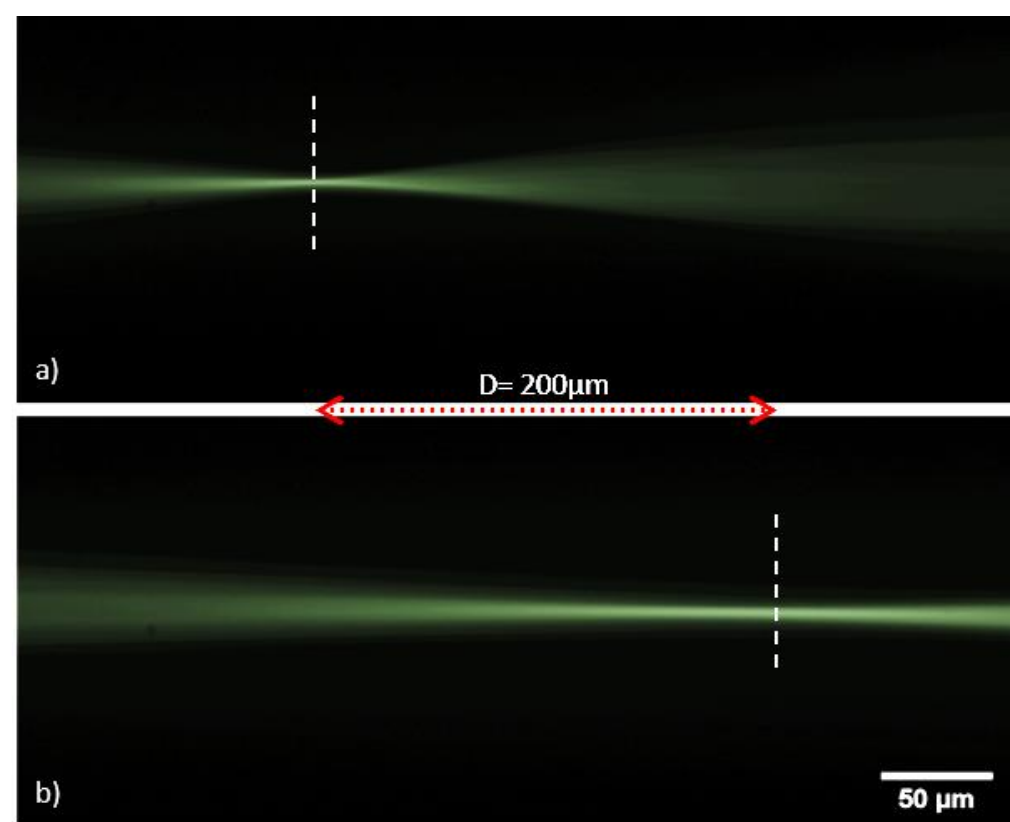

Figure 5. (a) Focused beam with unactuated VFM mirror at original position. (b) VFM mirror actuated with $\mathrm{V}_{\text {varifocal }}=15 \mathrm{~V}$ shifting the focus point by $200 \mu \mathrm{m}$.

The optical resolution of the imaging side was evaluated with the aid of a USAF 1951 optical target. The target is chromeprinted on a microscope slide and positioned at a $30^{\circ}$ angle in front of the imaging path. According to the slanted edge test and reconstruction of the Line Spread Function the experimentally achieved resolution was $0.95 \mu \mathrm{m}$. In order to quantify the focal plane change given by the tunable lens, images of different focal depths from the target were recorded as is shown in Figure 6. The angled placement of the target allows parts of the images to be focused during the three different power inputs. A total focal change of $200 \mu \mathrm{m}$ can be addressed with the tunable lens with input voltages in the range of $-0.5 \mathrm{~V}$, $0 \mathrm{~V},+0.5 \mathrm{~V}$ of the maximum $1 \mathrm{~V}$ specification of the lens. As a result of staying in the low actuation range of the lens, the spherical aberrations introduced by the tunable lens affect the imaging only minimal and are negligible. Furthermore, the field of view was measured at $250 \mu \mathrm{m}$ by $400 \mu \mathrm{m}$.
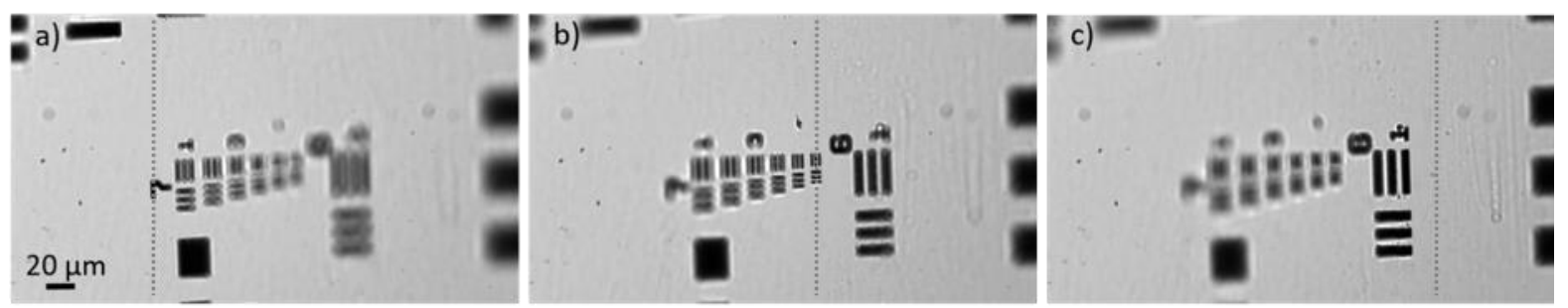

Figure 6. Imaging of angled USAF 1951 target group 7 for three different actuations of the tunable lens. Dotted vertical line indicates where the focal point of the image is located. (a) $\mathrm{x}=-100 \mu \mathrm{m}$ with $\mathrm{V}_{\text {tunable }}=-0.5 \mathrm{~V}$, (b) $\mathrm{x}=0 \mu \mathrm{m}$ with $\mathrm{V}_{\text {tunable }}=0 \mathrm{~V}$, (c) $\mathrm{x}=100 \mu \mathrm{m}$ with $\mathrm{V}_{\text {tunable }}=0.5 \mathrm{~V}$. 


\section{FLUORESCENCE IMAGING RESULTS}

\subsection{Imaging of different focal planes}

The fluorescence imaging capabilities for the LSM were evaluated with a FocalCheck microscope slide (Thermofisher FocalCheck Fluorescence Microscope Test Slide \#1). The microscope slide is placed at a $30^{\circ}$ angle, as is shown in the schematic in Figure 1. The geometry of the microscope slide placement allows imaging of different bead areas with synchronized movement of the PZT mirror and tunable lens in order to align the imaging focal plane with the corresponding light-sheet position. For demonstration purposes the largest beads available in the slide were used. The beads are ring stained with $15 \mu \mathrm{m}$ diameter. The first image displayed in Figure 7(a) shows a section of the $15 \mu \mathrm{m}$ bead with both the tunable lens input voltage and static actuation of the mirror at rest $\left(\mathrm{V}_{\text {tunable }}=0 \mathrm{~V}, \mathrm{~V}_{\text {static }}=0 \mathrm{~V}\right)$. In order to access the two beads located at $\mathrm{x}=45 \mu \mathrm{m}$, an input voltage of $\mathrm{V}_{\text {static }}=6.4 \mathrm{~V}$ will be applied to the PZT mirror. The light-sheet will now excite the fluorescence at the location of the two beads and a synchronized input of $\mathrm{V}_{\text {tunable }}=0.1 \mathrm{~V}$ will bring the section in focus as is shown in Figure 7(b).

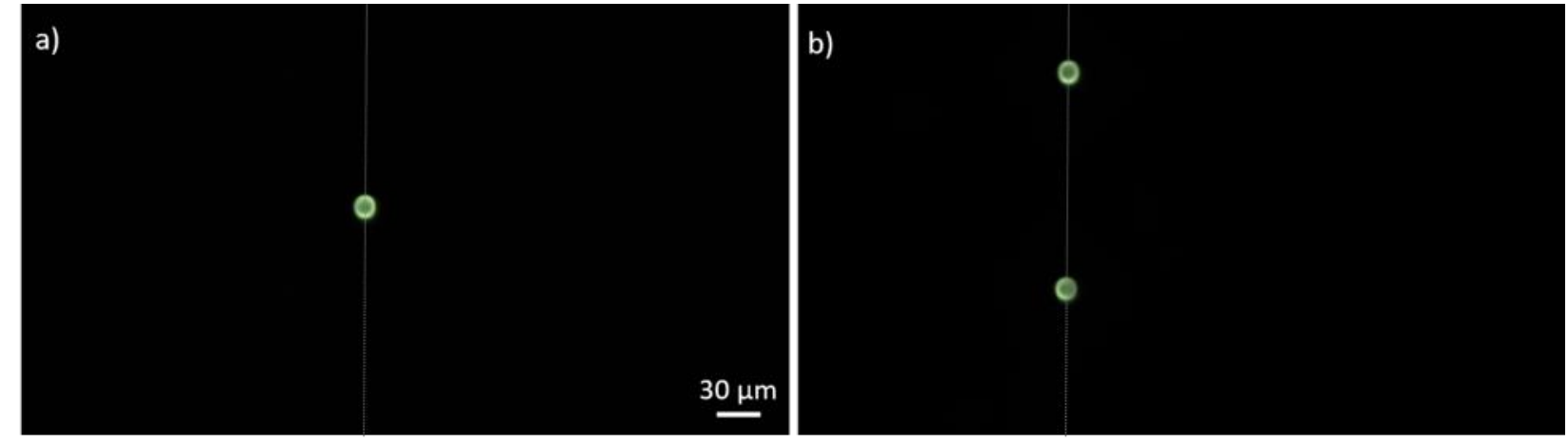

Figure 7. $15 \mu \mathrm{m}$ bead images captured at two different positions of (a) $\mathrm{x}=0 \mu \mathrm{m}$ and (b) $\mathrm{x}=45 \mu \mathrm{m}$. The beads are fixed on a microscope slide with only one thin layer being visible each time due to angled placement. Dotted vertical line indicates the focal point of the images.

\subsection{Fluorescence Imaging resolution estimation}

Further evaluation of fluorescent imaging was accomplished with the smallest beads of the target slide. The $1 \mu \mathrm{m}$ beads were chosen to evaluate the capability of visualising fluorescence targets near the resolution limit. As is shown in Figure 8(a) the beads are clearly visible as point like sources in the image. An exemplary profile plot of one of the beads is shown in Figure 8(b), which is closely matching the point spread function of the imaging capability.
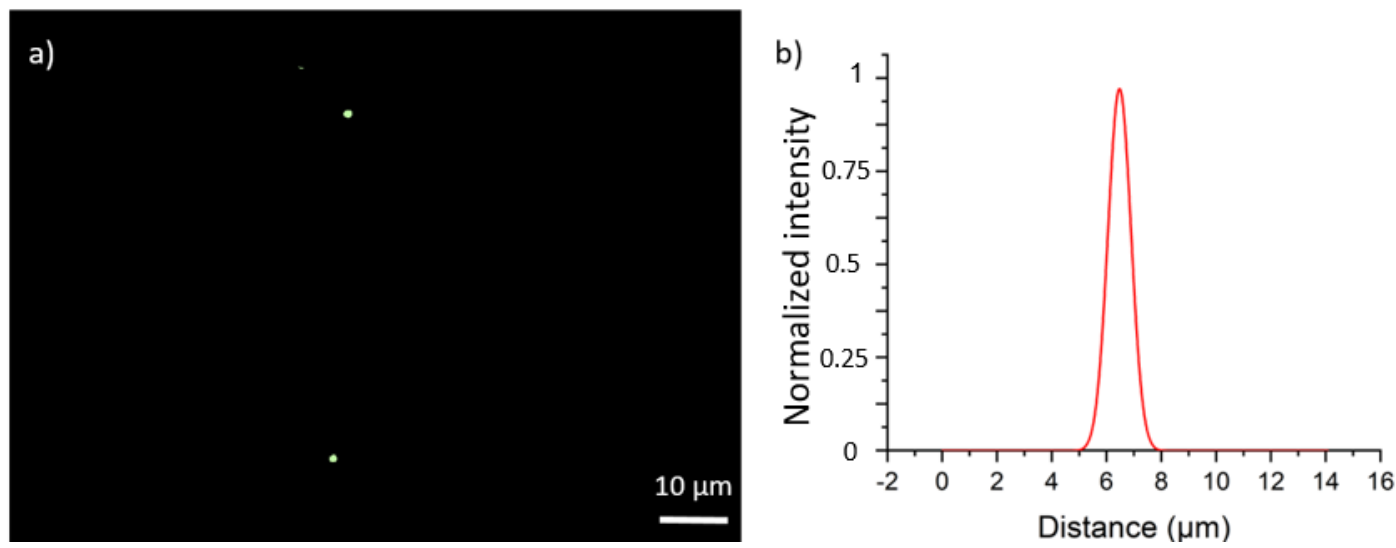

Figure 8. (a) Two $1 \mu \mathrm{m}$ beads in a zoomed in part of the overall FOV, (b) profile plot take from a single bead confirming resolution capabilities of $1 \mu \mathrm{m}$ fluorescent targets. 


\section{CONCLUSION}

The implementation of a miniaturized LSM system with active optical control was shown. The system was built to accomplish a cost and size effective solution with further control capabilities that allow light-sheet generation and repositioning coming from a PZT MEMS mirror and extension of laser beam focusing with the use of a VFM MEMS mirror. Additionally, a synchronized tunable lens in the imaging path was used to perform imaging of different focal planes. The results of the system with imaging both an optical target and a selection of fluorescent beads showed that the microscope can achieve sub-micron resolution as well as high intensity fluorescent sectioning in different focal planes.

Future implementations of the system will look at imaging cell samples fixed on both microscope slides and well plates. Additionally, synchronization of the VFM MEMS with the rolling shutter of the sCMOS camera will allow photobleaching reduction and increased contrast imaging.

\section{REFERENCES}

[1] Colombelli, J. and Lorenzo, C., 2014. "Light sheet fluorescence microscopy applications for multicellular systems" In Fluorescence Microscopy (pp. 109-120). Academic Press.

[2] Girkin, J. M. and Carvalho, M. T., "The light-sheet microscopy revolution,” J. Opt. 20(5), 053002 (2018).

[3] Huisken, J., Swoger, J., Del Bene, F., Wittbrodt, J. and Stelzer, E. H. K., "Optical sectioning deep inside live embryos by selective plane illumination microscopy," Science 305(5686), 1007-1009 (2004).

[4] Power, R. M. and Huisken, J., "A guide to light-sheet fluorescence microscopy for multiscale imaging," Nature Methods 14, 360-373 (2017).

[5] Meinert, T. and Rohrbach, A., "Light-sheet microscopy with length-adaptive Bessel beams," Biomedical Optics Express, 10(2), 670-681 (2019).

[6] Gustavsson, A.K., Petrov, P.N., Lee, M.Y., Shechtman, Y. and Moerner, W.E., “3D single-molecule superresolution microscopy with a tilted light sheet," Nature Communications, 9(1), 123 (2018).

[7] Landry, J.R., Itoh, R., Li, J.M., Hamann, S.S., Mandella, M., Contag, C.H. and Solgaard, O., "Tunable structured illumination light sheet microscopy for background rejection and imaging depth in minimally processed tissues," Journal of Biomedical Optics, 24(4), 046501 (2019).

[8] Zanacchi, F.C., Lavagnino, Z., Donnorso, M.P., Del Bue, A., Furia, L., Faretta, M. and Diaspro, A., "Live-cell 3D super-resolution imaging in thick biological samples," Nature methods, 8(12), 1047-1049 (2011).

[9] Hedde, P.N. and Gratton, E., "Selective plane illumination microscopy with a light sheet of uniform thickness formed by an electrically tunable lens," Microscopy research and technique, 81(9), 924-928 (2018).

[10]Ping, J., Zhao, F., Nie, J., Yu, T., Zhu, D., Liu, M. and Fei, P., "Propagating-path uniformly scanned light sheet excitation microscopy for isotropic volumetric imaging of large specimens," Journal of Biomedical Optics, 24(8), 086501 (2019).

[11] Vettenburg, T., Dalgarno, H.I., Nylk, J., Coll-Lladó, C., Ferrier, D.E., Čižmár, T., Gunn-Moore, F.J. and Dholakia, K., "Light-sheet microscopy using an Airy beam," Nature Methods, 11(5), 541-544 (2014).

[12] Fahrbach, F.O., Gurchenkov, V., Alessandri, K., Nassoy, P. and Rohrbach, A., "Light-sheet microscopy in thick media using scanned Bessel beams and two-photon fluorescence excitation," Optics Express, 21(11), 1382413839 (2013).

[13] Kashekodi, A.B., Meinert, T., Michiels, R. and Rohrbach, A., "Miniature scanning light-sheet illumination implemented in a conventional microscope," Biomedical Optics Express, 9(9), 4263-4274 (2018).

[14] Marx, V., "Microscopy: OpenSPIM 2.0,” Nature Methods, 13(12), 979-982 (2016).

[15] Bakas, S., Uttamchandani, D., Toshiyoshi, H. and Bauer, R., "MEMS Enabled Control of Light-Sheet Microscopy Optical Beam Paths," 2018 Int. Conf. Opt. MEMS Nanophotonics, 145-146, IEEE, Lausanne, Switzerland (2018) 